\title{
Investigation of microcirculation in patients with venoarterial extracorporeal membrane oxygenation life support
}

Yu-Chang Yeh', Chen-Tse Lee ${ }^{1}$, Chih-Hsien Wang ${ }^{2}$, Yu-Kang Tu ${ }^{3}$ Chien-Heng Lai ${ }^{2}$, Yin-Chin Wang ${ }^{1}$, Anne Chao ${ }^{1}$, Chi-Hsiang Huang ${ }^{1}$, Ya-Jung Cheng ${ }^{1 *+}$, Yih-Sharng Chen ${ }^{2^{*+}}$ and on behalf of the NTUH Center of Microcirculation Medical Research (NCMMR)

\begin{abstract}
Background: Microcirculatory dysfunction develops in both septic and cardiogenic shock patients, and it is associated with poor prognosis in patients with septic shock. Information on the association between microcirculatory dysfunction and prognosis in cardiogenic shock patients with venoarterial extracorporeal membrane oxygenation (VA-ECMO) support is limited.
\end{abstract}

Methods: Sublingual microcirculation images were recorded using an incident dark-field video microscope at the following time points: within $12 \mathrm{~h}$ (T1), $24 \mathrm{~h}$ (T2), $48 \mathrm{~h}$ (T3), $72 \mathrm{~h}$ (T4), and $96 \mathrm{~h}$ (T5) after VA-ECMO placement. If a patient could be weaned off $\mathrm{VA}-\mathrm{ECMO}$, sublingual microcirculation images were recorded before and after VA-ECMO removal. Microcirculatory parameters were compared between 28-day nonsurvivors and survivors with VA-ECMO support. In addition, the microcirculation and clinical parameters were assessed as prognostic tests of 28-day mortality, and patients were divided into three subgroups according to microcirculation parameters for survival analysis.

Results: Forty-eight patients were enrolled in this study. At T1, the observed heart rate, mean arterial pressure, inotropic score and lactate level of 28-day nonsurvivors and survivors did not differ significantly, but the perfused small vessel density (PSVD) and proportion of perfused vessels (PPV) were lower in the 28-day nonsurvivors than in the survivors. The PSVD and PPV were slightly superior to lactate levels in predicting 28-day mortality (area under curve of $0.68,0.70$, and 0.62 , respectively). The subgroup with the lowest PSVD $\left(<15 \mathrm{~mm} / \mathrm{mm}^{2}\right)$ and PPV $(<64 \%)$ values exhibited less favorable survival compared with the other two subgroups.

Conclusions: Early microcirculatory parameters could be used to predict the survival of cardiogenic shock patients with VA-ECMO support.

Trial registration: ClinicalTrials.gov, NCT02393274. Registered on 19 March 2015.

Keywords: Cardiogenic shock, Extracorporeal membrane oxygenation, Microcirculation, Survival

\footnotetext{
* Correspondence: chengyj@ntu.edu.tw; yschen1234@gmail.com

†Ya-Jung Cheng and Yih-Sharng Chen contributed equally to this work.

'Department of Anesthesiology, National Taiwan University Hospital, College

of Medicine, National Taiwan University, No 7, Chung Shang South Road,

Taipei, Taiwan

2Department of Surgery, National Taiwan University Hospital, College of

Medicine, National Taiwan University, No 7, Chung Shang South Road, Taipei,

Taiwan

Full list of author information is available at the end of the article
}

(c) The Author(s). 2018 Open Access This article is distributed under the terms of the Creative Commons Attribution 4.0 International License (http://creativecommons.org/licenses/by/4.0/), which permits unrestricted use, distribution, and reproduction in any medium, provided you give appropriate credit to the original author(s) and the source, provide a link to the Creative Commons license, and indicate if changes were made. The Creative Commons Public Domain Dedication waiver (http://creativecommons.org/publicdomain/zero/1.0/) applies to the data made available in this article, unless otherwise stated. 


\section{Background}

An extracorporeal membrane oxygenation (ECMO) life support system can provide both cardiac and respiratory support to patients with heart failure, respiratory failure, or both [1-4]. It can afford time for the failed organs to recover or for the patients to receive further management. However, many patients can possibly die despite ECMO support. One of the key factors is whether the blood flow provided by the ECMO system can restore organ perfusion. The adequacy of macrocirculation may be determined by arterial pressure and the minute blood flow of venoarterial ECMO (VA-ECMO). However, the adequacy of the microcirculation remains a major unresolved clinical concern in patients with ECMO. Microcirculatory dysfunction has been observed in patients who have suffered septic shock or cardiogenic shock, and in patients who have undergone surgery [5-9]. Moreover, microcirculatory dysfunction is associated with poor prognosis in patients with severe sepsis and in patients who have suffered an out-of-hospital cardiac arrest [10-12]. Because information regarding the association between microcirculatory dysfunction and prognosis in patients with VA-ECMO support is limited [13], this study focused on comparing perfused small vessel density (PSVD) between 28-day nonsurvivors and survivors by visualizing their microcirculation using a third-generation video microscope within $12 \mathrm{~h}$ after VA-ECMO placement [14, 15]. In addition, the microcirculation and clinical parameters were assessed as prognostic tests of 28-day mortality, and the patients were divided into three subgroups according to microcirculation parameters for survival analysis.

\section{Methods}

\section{Patients}

This prospective observational study was approved by the Research Ethics Committee of National Taiwan University Hospital (approval number 201412045RINA) and registered on the ClinicalTrials.gov protocol registration system (NCT02393274). This study was conducted at National Taiwan University Hospital between June 2015 and August 2016. Participants for the study were selected from patients receiving ECMO support; they were screened and evaluated for eligibility within $12 \mathrm{~h}$ after ECMO placement. The inclusion criterion was that patients should have suffered from cardiogenic shock and received VA-ECMO support. Patients who were aged < 20 or $>80$ years, those for whom sublingual microcirculation could not be measured within $12 \mathrm{~h}$ after VA-ECMO placement (i.e., placement occurred in the evening, during holidays, or when the research assistant was on leave), and those who were non-native speakers were excluded. Informed consent of the patients was obtained from their legally authorized representatives before enrollment in the study. Sublingual microcirculation images were recorded using an incident dark-field video microscope (CytoCam, Braedius Medical, Huizen, the Netherlands) [16]. The images were recorded at the following time points: within $12 \mathrm{~h}$ (T1), $24 \mathrm{~h}$ (T2), $48 \mathrm{~h}$ (T3), $72 \mathrm{~h}$ (T4), and $96 \mathrm{~h}$ (T5) after VA-ECMO placement. If the patients could be weaned off VA-ECMO support, sublingual microcirculation images were recorded at the following time points: before removal (R0), and $6 \mathrm{~h}$ (R1), $24 \mathrm{~h}$ (R2), $48 \mathrm{~h}$ (R3), and $72 \mathrm{~h}$ (R4) after VA-ECMO removal.

\section{VA-ECMO components and placement}

For all enrolled patients, the VA-ECMO was placed in the femoral artery and vein using the cut-down method. The principal component of VA-ECMO included a heparin-bonded surface circuit, a centrifugal pump (BPX-80 Bio-Pump Plus, Medtronic, Anaheim, CA, USA), an oxygenator (Affinity NT, Medtronic), an oxygen-air blender (Model 3500 CP-G gas mixer, Sechrist, Anaheim, CA, USA), and a cannula (BE-HLS, Maquet, Turkey). To avoid possible malperfusion of the distal limb, an antegrade distal perfusion catheter was used when the mean pressure of the superficial femoral artery was below $50 \mathrm{mmHg}$ [17]. All patients received standard management of VA-ECMO and routine intensive care unit (ICU) care. Data pertaining to the following parameters were recorded: age, gender, height, body weight, Acute Physiology and Chronic Health Evaluation (APACHE) II score, Sequential Organ Failure Assessment (SOFA) score [18], indications of VA-ECMO, VA-ECMO blood flow, heart rate, mean arterial pressure (MAP), lactate level, activated clotting time, hemoglobin, fluid balance, and inotropic score. The inotropic score was calculated as $100 \times$ epinephrine dose $(\mu \mathrm{g} / \mathrm{kg} / \mathrm{min})+$ $100 \times$ norepinephrine dose $(\mu \mathrm{g} / \mathrm{kg} / \mathrm{min})+$ dopamine dose $(\mu \mathrm{g} / \mathrm{kg} / \mathrm{min})+$ dobutamine dose $(\mu \mathrm{g} / \mathrm{kg} / \mathrm{min})$ [19]. The use of intra-aortic balloon pump or continuous arteriovenous hemofiltration was recorded if simultaneously used with VA-ECMO support. The length of VA-ECMO support, length of ICU and hospital stay, and survival status at 28 days were also recorded. Heparin was continuously infused to maintain an activated clotting time of 160-180 s if no active bleeding or other complications were observed.

\section{Measurements of sublingual microcirculation}

At each time point, five video sequences (time length $6 \mathrm{~s}$ ) were recorded from different sublingual sites and were digitally stored with code numbers to ensure blinding of patient information. Subsequent offline analyses were performed by a single observer who was blinded to the patient information. The most appropriate three sequences were selected for analysis using the semi-automated 
analysis software package Automated Vascular Analysis (AVA) 3.0 (Academic Medical Center, University of Amsterdam, Amsterdam, the Netherlands). According to the suggestions of a previously held roundtable conference for evaluating the microcirculation [20], the following parameters were investigated: a) total small vessel (less than $20 \mu \mathrm{m})$ density (TSVD); b) perfused small vessel density (PSVD); c) proportion of perfused vessels (PPV); d) microvascular flow index (MFI) score; and e) heterogeneity index (HI). The TSVD was automatically calculated by the software. The blood flow in small vessels was semiquantitatively classified using an ordinal scale of $0-3$ in accordance with the methods described in our previous study [5]. Small vessels with a blood flow classification of 2 or 3 were considered perfused vessels, and the PSVD was automatically calculated by the software. The MFI score and HI were semiquantitatively calculated according to the suggestions of the roundtable conference [20]. The primary endpoint was determining the difference between PSVD of 28-day survivors and nonsurvivors at T1. Based on our experience, 20 patients per group is sufficient to detect a $17.5 \%$ difference of PSVD between the two groups, with an $\alpha$ level of 0.05 (two-tailed) and a $\beta$ level of 0.2 , assuming a control mean PSVD of $20 \mathrm{~mm} / \mathrm{mm}^{3}$ with a standard deviation of 4 .

\section{Prognostic tests of 28-day mortality and subgroup survival analysis}

Receiver operating characteristic (ROC) curves and the corresponding area under the curve (AUC) were used for assessing the discriminative abilities of APACHE II score, lactate level, PSVD, and PPV at T1 for 28-day mortality. Cutoff points were calculated by obtaining the optimal Youden index (sensitivity + specificity -1 ). Moreover, patients were divided into three groups according to the 25th and 75th percentiles of PSVD and PPV values for the 28-day survival analysis among the three groups.

\section{Statistical analysis}

Data were analyzed using the statistical software SPSS 20 (IBM, Armonk, NY, USA). Normally distributed numerical data are expressed as mean (standard deviation), and data for 28-day survivors and nonsurvivors were compared using $t$ tests. Non-normally distributed numerical data and the MFI score are expressed as median (interquartile range), and data for 28-day survivors and nonsurvivors were compared using the Mann-Whitney test. Categorical variables are described as a percentage and were compared using the chi-square test or Fisher's exact test, as appropriate. A $p$ value $<0.05$ was considered significant.

\section{Results}

\section{Patient characteristics}

A total of 246 patients receiving VA-ECMO support were screened for determining their eligibility for this trial. In total, 45 patients receiving venovenous ECMO did not meet the inclusion criterion, and 153 patients were excluded (Fig. 1). Therefore, 48 patients were enrolled in this study, and the 28-day survival rate was $50 \%$. Values for the baseline characteristics, indications of VA-ECMO, APACHE II score, SOFA score, fluid balance, use of intra-aortic balloon pump or continuous arteriovenous hemofiltration, number of patients who underwent heart transplantation, number of discharged patients from 28-day survivors, length of ICU stay, and length of hospital stay are presented in Table 1.

Hemodynamic parameters, inotropic score, lactate level, and microcirculatory parameters at different time points Values for the hemodynamic parameters, inotropic score, lactate level, and microcirculatory parameters at $\mathrm{T} 1, \mathrm{~T} 2$, T3, T4, and T5 are shown in Fig. 2 and Table 2. At T1, the observed MAP, inotropic score, and lactate level did not differ significantly between the 28-day nonsurvivors and survivors, but the PSVD and PPV for the 28-day nonsurvivors were lower than those for the survivors. Values for the hemodynamic parameters, inotropic score, lactate level, and microcirculatory parameters at R0, R1, R2, R3, and $\mathrm{R} 4$ are presented in Fig. 3. At R0, the MFI score did not differ between the 28-day nonsurvivors and survivors; by contrast, at R1, the MFI score for the 28-day nonsurvivors was lower than that for the survivors. .

\section{Prognostic tests and subgroup survival analysis of 28-day mortality}

The ROC curves for prognostic tests of 28-day mortality are illustrated in Fig. 4. According to the ROC curve

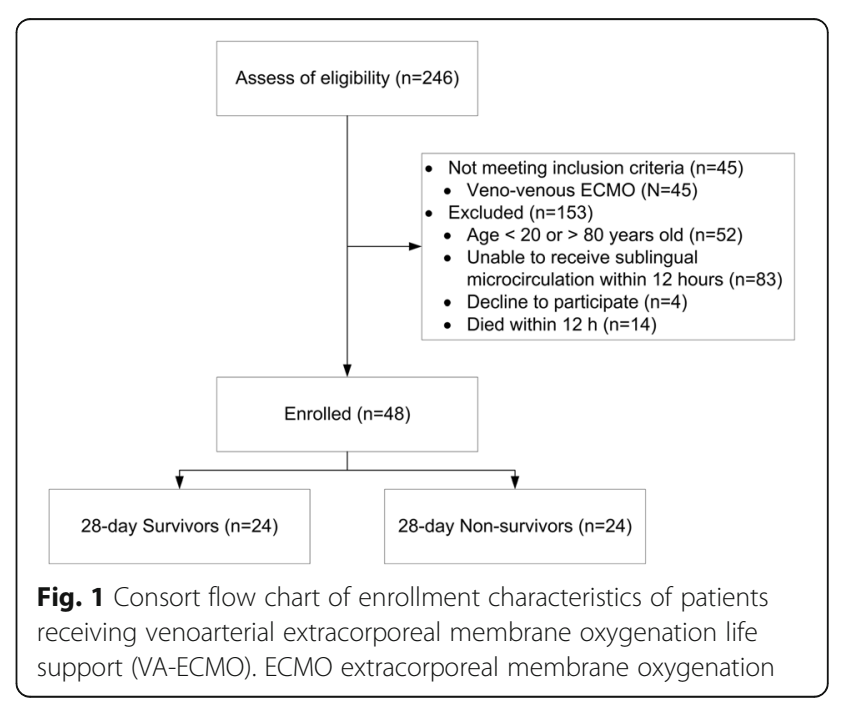


Table 1 Patient characteristics

\begin{tabular}{|c|c|c|c|}
\hline Characteristics & $\begin{array}{l}\text { 28-day survivors } \\
(n=24)\end{array}$ & $\begin{array}{l}\text { 28-day nonsurvivors } \\
(n=24)\end{array}$ & $p$ \\
\hline Age (years) & $53(13)$ & $60(12)$ & 0.050 \\
\hline Female/male & $6 / 18$ & $7 / 17$ & 0.745 \\
\hline Body weight (kg) & $69(17)$ & $68(14)$ & 0.773 \\
\hline Height (cm) & $168(10)$ & $161(22)$ & 0.272 \\
\hline \multicolumn{4}{|l|}{ Indication of ECMO } \\
\hline Heart failure & 12 & 10 & \\
\hline Postcardiotomy & 1 & 1 & \\
\hline ECPR & 10 & 11 & \\
\hline Septic shock & 0 & 2 & \\
\hline ARDS with shock & 1 & 0 & \\
\hline APACHE II_T1 & $19(7)$ & $26(8)$ & 0.002 \\
\hline SOFA_T1 & $13(3)$ & $14(3)$ & 0.208 \\
\hline APACHE II_T2 & $17(7)$ & $24(9)$ & 0.005 \\
\hline SOFA_T2 & $13(4)$ & $15(3)$ & 0.049 \\
\hline APACHE II_RO & $15(6)$ & $20(6)$ & 0.014 \\
\hline SOFA_RO & $11(3)$ & $16(3)$ & $<0.001$ \\
\hline Fluid balance $6 \mathrm{~h}(\mathrm{ml})$ & 1019 (2016) & $2801(3210)$ & 0.026 \\
\hline Fluid balance $24 \mathrm{~h}(\mathrm{ml})$ & $266(1221)$ & 1955 (3027) & 0.022 \\
\hline Fluid balance $48 \mathrm{~h}(\mathrm{ml})$ & $-44(922)$ & 953 (2184) & 0.104 \\
\hline IABP_T1, n (\%) & $8(33 \%)$ & $6(25 \%)$ & 0.752 \\
\hline CAVH_T1, n (\%) & $6(25 \%)$ & $12(50 \%)$ & 0.074 \\
\hline CAVH_T1-T5, n (\%) & $12(50 \%)$ & $18(75 \%)$ & 0.074 \\
\hline Heart transplant, $n(\%)$ & $3(13 \%)$ & $1(4 \%)$ & 0.609 \\
\hline Length of ECMO support (day) & $5(3-8.5[1-54])$ & & \\
\hline Survive to discharge & $n=22$ & & \\
\hline ICU stay (day) & $18(8-41[4-70])$ & & \\
\hline Hospital stay (day) & 39 (22-70 [11-127]) & & \\
\hline
\end{tabular}

Data are presented as mean (standard deviation), $n$ (\%), or median (interquartile range [range])

$\mathrm{T} 1, \mathrm{~T} 2$, and $\mathrm{T} 5$ represent within 12, 24, and 96 h, respectively, after placement of extracorporeal membrane oxygenation life support system (ECMO) $\mathrm{RO}$ represents before removal of ECMO

APACHE Acute Physiology and Chronic Health Evaluation, ARDS acute respiratory distress syndrome, $C A V H$ continuous arteriovenous hemofiltration, $E C P R$ extracorporeal cardiopulmonary resuscitation, IABP intra-aortic balloon pump, ICU intensive care unit, SOFA Sequential Organ Failure Assessment

analysis, the threshold values of the APACHE II score, lactate level, PSVD, and PPV were 22.5, $7.5 \mathrm{mmol} / \mathrm{l}$, $16.2 \mathrm{~mm} / \mathrm{mm}^{2}$, and $76.5 \%$, respectively. The 28 -day survival curves based on the subgrouping according to PSVD and PVD values at T1 are presented in Fig. 5. The patients in the two subgroups with higher PSVD and PPV values exhibited greater survival than those in the subgroup with the lowest PSVD and PPV values.

\section{Discussion}

This prospective observational study showed that microcirculatory dysfunction was more severe in 28-day nonsurvivors than in survivors with VA-ECMO support. Moreover, this study revealed that the PSVD and PPV at T1 could be used to predict the survival of such patients.
Furthermore, when the patients were divided into three subgroups according to the 25th and 75th percentiles of PSVD and PPV values at T1, the patients in the two subgroups with higher PSVD and PPV values exhibited greater survival than those in the subgroup with the lowest PSVD and PPV values.

The main finding of this study is that the PSVD and PPV at T1 in the 28-day nonsurvivors were lower than in the survivors, but the observed MAP, inotropic score, and lactate level at $\mathrm{T} 1$ did not differ significantly between the 28-day nonsurvivors and survivors. This disparity was consistent with the notion that microcirculatory dysfunction can occur in normal macrocirculation parameters [21, 22]. Therefore, we suggest that MAP might not be suitable as an optimal or final resuscitation goal for 


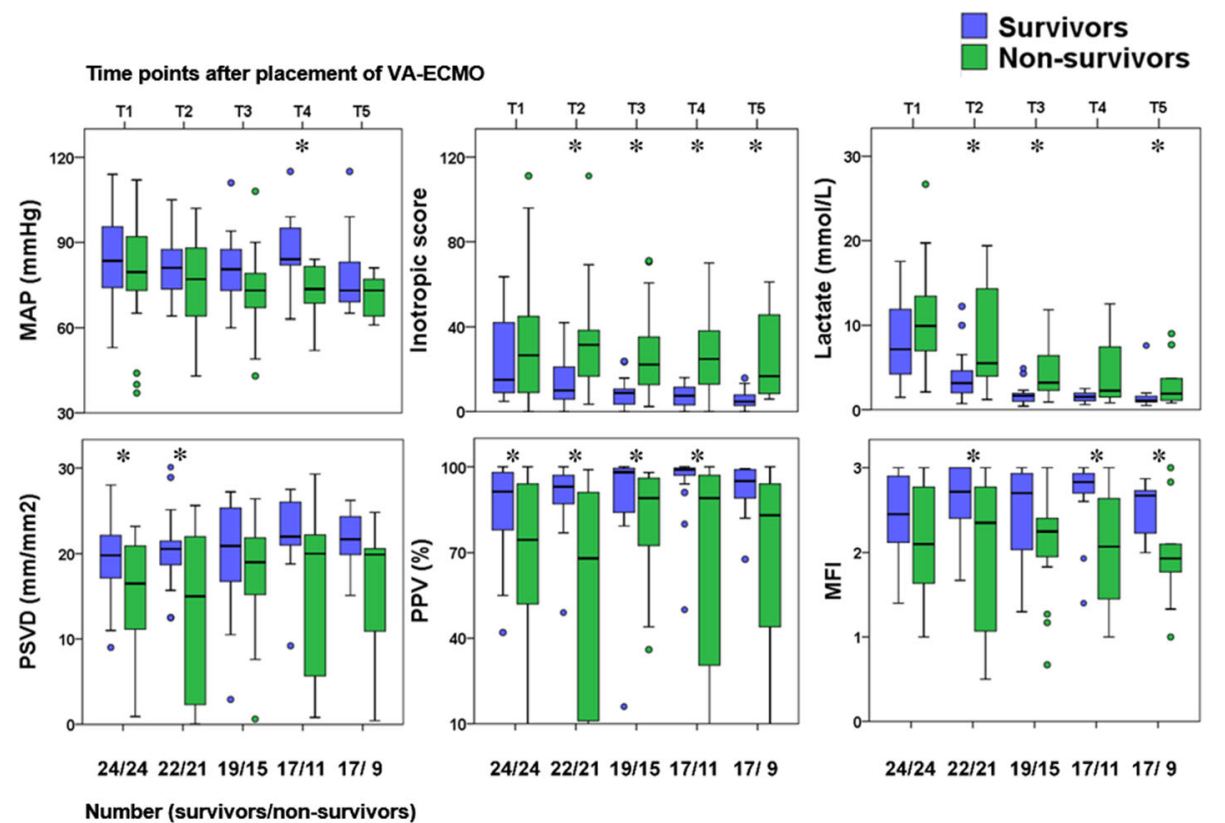

Fig. 2 Mean arterial pressure (MAP), inotropic score, lactate level, and microcirculation parameters of 28-day survivors and nonsurvivors after placement of venoarterial extracorporeal membrane oxygenation life support (VA-ECMO). The time points after placement of VA-ECOM are presented as T1 (within 12 h), T2 (24 h), T3 (48 h), T4 (72 h), and T5 (96 h). ${ }^{*} p<0.05$ between 28-day survivors and nonsurvivors. MFI microvascular flow index, PPV proportion of perfused vessels, PSVD perfused small vessel density

Table 2 ECMO blood flow and microcirculatory parameters of 28-day survivors and nonsurvivors at T1, T2, T3, T4, and T5

\begin{tabular}{|c|c|c|c|}
\hline Parameters & 28-day survivors & 28-day nonsurvivors & $p$ \\
\hline $\mathrm{T} 1$ (within $12 \mathrm{~h}$ after ECMO placement) & $(n=24)$ & $(n=24)$ & \\
\hline ECMO blood flow (L/min) & $2.3(0.8)$ & $2.5(0.6)$ & 0.191 \\
\hline $\operatorname{TSVD}\left(\mathrm{mm} / \mathrm{mm}^{2}\right)$ & $22.5(2.7)$ & $22.0(3.5)$ & 0.576 \\
\hline $\mathrm{HI}$ & $0.30(0.1-0.44)$ & $0.38(0.09-0.71)$ & 0.909 \\
\hline T2 (24 h after ECMO placement) & $(n=22)$ & $(n=21)$ & \\
\hline ECMO blood flow (L/min) & $2.2(0.4)$ & $2.5(0.5)$ & 0.022 \\
\hline $\operatorname{TSVD}\left(\mathrm{mm} / \mathrm{mm}^{2}\right)$ & $22.8(3.6)$ & $22.8(3.4)$ & 0.994 \\
\hline $\mathrm{HI}$ & $0.21(0-0.41)$ & $0.24(0.18-0.57)$ & 0.269 \\
\hline T3 (48 h after ECMO placement) & $(n=19)$ & $(n=15)$ & \\
\hline ECMO blood flow (L/min) & $2.1(0.4)$ & $2.5(0.9)$ & 0.176 \\
\hline $\operatorname{TSVD}\left(\mathrm{mm} / \mathrm{mm}^{2}\right)$ & $21.7(4.4)$ & $22.7(3.4)$ & 0.442 \\
\hline $\mathrm{HI}$ & $0.2(0.07-0.3)$ & $0.33(0.14-0.44)$ & 0.040 \\
\hline T4 (72 h after ECMO placement) & $(n=17)$ & $(n=11)$ & \\
\hline ECMO blood flow (L/min) & $2.2(0.4)$ & $2.8(1.2)$ & 0.097 \\
\hline $\operatorname{TSVD}\left(\mathrm{mm} / \mathrm{mm}^{2}\right)$ & $23.8(3.4)$ & $21.8(4.5)$ & 0.177 \\
\hline $\mathrm{HI}$ & $0.14(0.05-0.25)$ & $0.18(0.05-0.32)$ & 0.353 \\
\hline T5 (96 h after ECMO placement) & $(n=17)$ & $(n=9)$ & \\
\hline ECMO blood flow (L/min) & $2.1(0.6)$ & $3.0(1.4)$ & 0.089 \\
\hline $\operatorname{TSVD}\left(\mathrm{mm} / \mathrm{mm}^{2}\right)$ & $23.3(2.9)$ & $21.3(4.8)$ & 0.183 \\
\hline $\mathrm{HI}$ & $0.31(0.1-0.54)$ & $0.18(0-0.56)$ & 0.458 \\
\hline
\end{tabular}




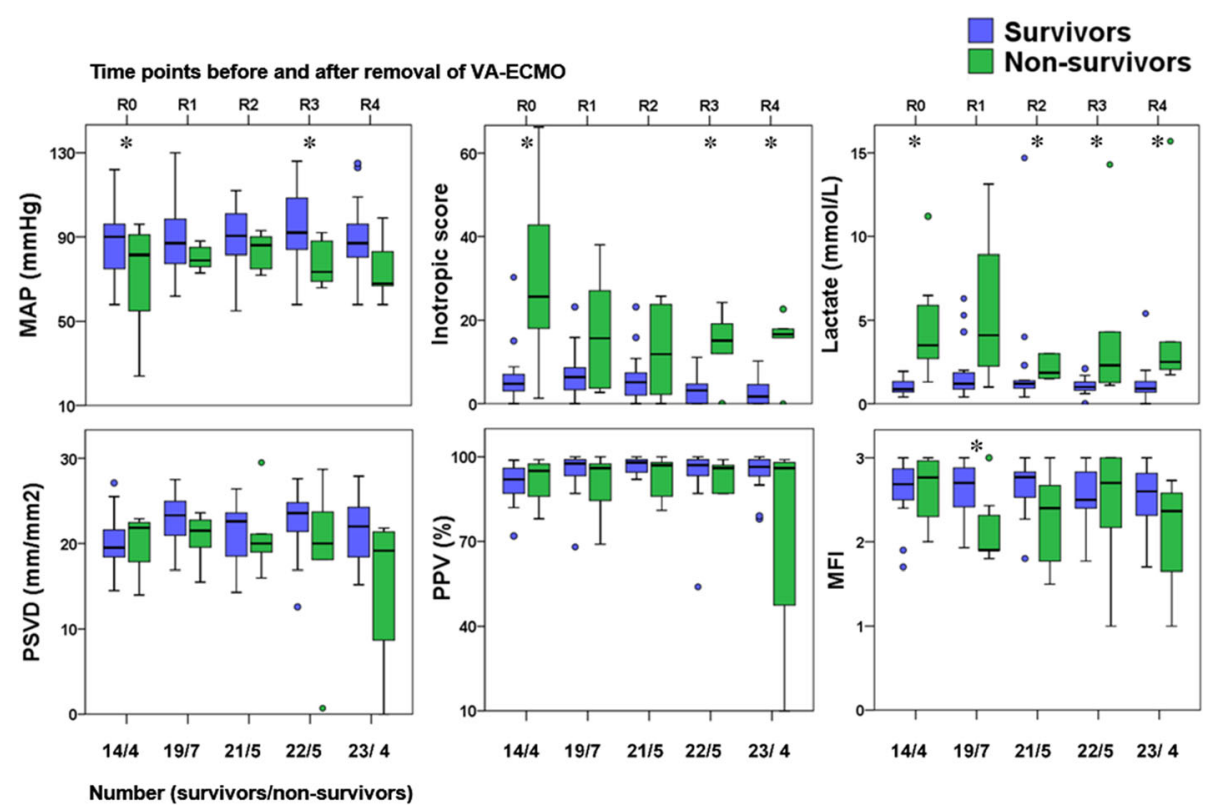

Fig. 3 Mean arterial pressure (MAP), inotropic score, lactate level, and microcirculation parameters of 28-day survivors and nonsurvivors before and after removal of venoarterial extracorporeal membrane oxygenation life support (VA-ECMO). The time point before removal of VA-ECOM is presented as R0, and the time points after removal of VA-ECOM are presented as R1 (6 h), R2 (24 h), R3 (48 h), and R4 (72 h). ${ }^{*} p<0.05$ between 28-day survivors and nonsurvivors. MFI microvascular flow index, PPV proportion of perfused vessels, PSVD perfused small vessel density

patients with cardiogenic shock receiving VA-ECMO support. However, measuring microcirculation parameters may help to predict outcomes and provide information on the adequacy of tissue perfusion. Further studies are required to investigate the effect of improving microcirculation on survival. In addition, fluid balance was higher in

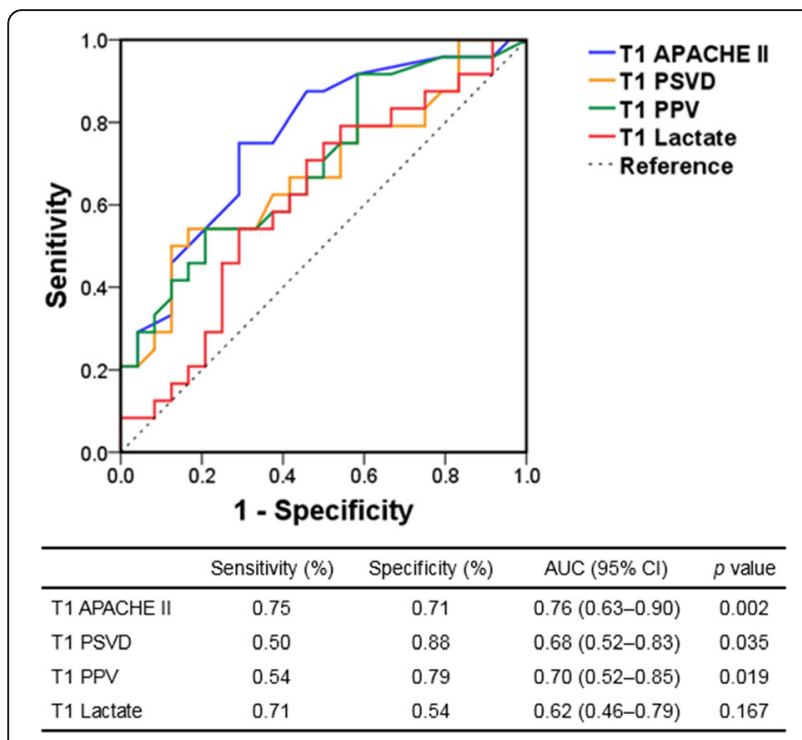

Fig. 4 Prognostic tests of 28-day mortality. APACHE Acute Physiology and Chronic Health Evaluation, AUC area under the curve, Cl confidence interval, PPV proportion of perfused vessels, PSVD perfused small vessel density the 28-day nonsurvivors than in the survivors. Fluid overload might have resulted from the higher severity of shock in the 28-day nonsurvivors. Such overload of fluid might result in increased diffusive distance of the small vessels, reducing the ability of oxygen to reach the tissue cells [23].

Our result that microcirculatory dysfunction was more severe in 28-day nonsurvivors than in survivors with VA-ECMO support agrees with the findings of Kara et al. [13]. However, there are several differences between the findings of the two studies. First, we found that the APACHE II score for the 28-day nonsurvivors was higher than that for the survivors. However, in the study by Kara et al., the APACHE II scores for 28-day nonsurvivors and survivors did not differ significantly. Second, the PPV value for both 28-day survivors and nonsurvivors in our study are lower than those reported by Kara et al. Third, in our study, the level of ECMO blood flow at T2 was higher in the 28-day nonsurvivors than in the survivors. However, in the study of Kara et al., the levels of ECMO blood flow in survivors and nonsurvivors did not differ significantly. In addition, the level of VA-ECMO blood flow in the study by Kara et al. is higher than that in our study. There are several explanations for the different findings of the two studies. First, we enrolled more patients in this study than did Kara et al. (48 vs 24 patients). Second, we measured baseline microcirculatory parameters within the first $12 \mathrm{~h}$ after VA-ECMO placement, whereas Kara et al. measured 

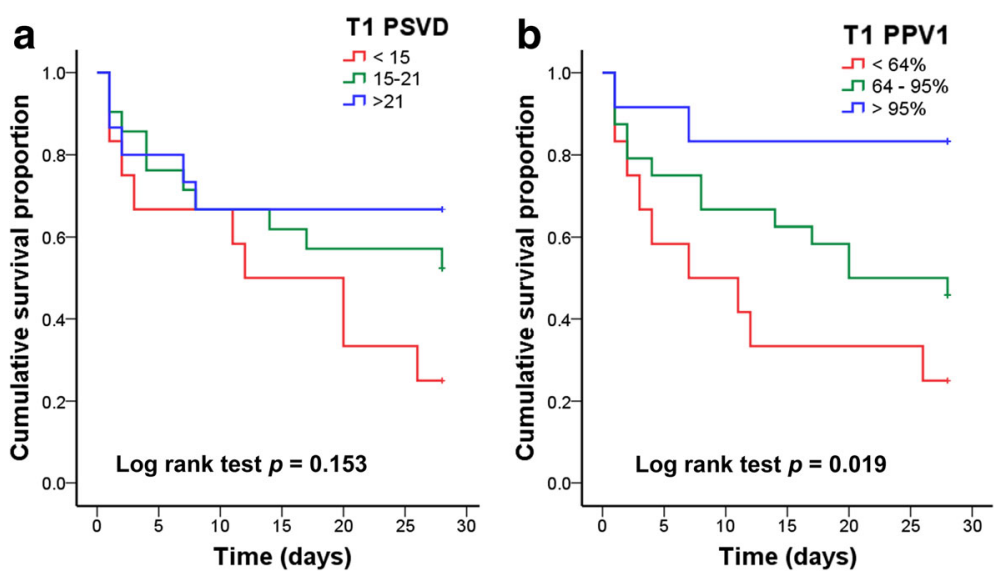

Fig. 5 Twenty-eight-day survival curves based on subgrouping by perfused small vessel density (PSVD) and proportion of perfused vessels (PPV). Patients were divided into three subgroups according to the 25 th and 75 th percentiles of a PSVD $\left(\mathrm{mm} / \mathrm{mm}^{2}\right)$ and $\mathbf{b}$ PPV (\%) values

these parameters within the first $24 \mathrm{~h}$. Third, the two studies had different definitions of diameter of small vessels $(<20 \mu \mathrm{m}$ vs $<25 \mu \mathrm{m})$. In addition, Kara et al. used the PSVD of all vessels $(<100 \mu \mathrm{m})$ to predict survival from ROC curves. Fourth, the two studies had different definitions of mortality (28-day mortality vs ICU mortality).

In our study, the PSVD derived for the 28-day survivors at R4 was still lower than that derived for 70 healthy volunteers in our unpublished study (21.8 (3.7) vs. $\left.25.2(2.3) \mathrm{mm} / \mathrm{mm}^{2}, p<0.001\right)$. Persistent microcirculatory dysfunction in patients with VA-ECMO support perhaps results from primary diseases, inflammatory response of VA-ECMO [24], and hemolysis-associated residual endothelium dysfunction [25, 26]. Persistent microcirculatory dysfunction is associated with organ failure and death in patients with septic shock [22]. Additional studies are required to investigate the effect of persistent microcirculatory dysfunction on organ dysfunction in patients with VA-ECMO support. The MFI scores for the 28-day nonsurvivors and survivors did not differ significantly before VA-ECMO removal. However, within $6 \mathrm{~h}$ after VA-ECMO removal, the MFI score for the 28-day nonsurvivors became lower than that for the survivors. Additional studies are required to investigate changes in MFI score while decreasing VA-ECMO blood flow before VA-ECMO removal; this may provide information to predict the microcirculation status following VA-ECMO removal. Additional studies may also compare the predictabilities of microcirculatory parameters with the current VA-ECMO weaning predictors [1, 27, 28]. Moreover, lactate levels were higher in the 28-day nonsurvivors than in the survivors at R0, R2, R3, and R4. Thus, lactate levels might provide further information before and after the removal of VA-ECMO.
This study had several limitations. First, the mechanism of microcirculatory dysfunction and its effects on mortality might vary in different primary etiologies of cardiogenic shock, but the sample size of this study was too small to investigate such variances. Second, the number of 28-day nonsurvivors decreased at other time points due to death after T1. Comparisons between the variables of 28-day nonsurvivors and survivors at other time points might not have had sufficient power to detect significant differences. In addition, this meant the trial was not suitable for a nonparametric analysis of variance (ANOVA) for repeated measures. These data provide preliminary information for further studies to investigate the microcirculation at other time points after VA-ECMO placement and VA-ECMO removal. Third, the optimal cutoff points of the microcirculatory parameters could have been influenced by the defined diameter of small vessels $(<20 \mu \mathrm{m}$ or $<25 \mu \mathrm{m}$ ) or the range of observed microcirculatory vessels (only small vessels or total vessels). We suggest that the different primary etiologies of cardiogenic shock might affect different types of microcirculatory vessels. Additional studies are required to investigate the optimal defined diameter of small vessels and the optimal range of observed microcirculatory vessels in different etiologies of cardiogenic shock.

\section{Conclusions}

We show that early microcirculatory parameters could be used to predict the survival of patients with cardiogenic shock with VA-ECMO support. Additional studies are required to investigate whether improving microcirculation can improve the survival of patients with cardiogenic shock receiving VA-ECMO support.

\section{Abbreviations}

ANOVA: Analysis of variance; APACHE: Acute Physiology and Chronic Health Evaluation; AUC: Area under the curve; AVA: Automated Vascular Analysis; 
ECMO: Extracorporeal membrane oxygenation; HI: Heterogeneity index; ICU: Intensive care unit; MAP: Mean arterial pressure; MFI: Microvascular flow index; PPV: Proportion of perfused vessels; PSVD: Perfused small vessel density; ROC: Receiver operating characteristic; SOFA: Sequential Organ Failure Assessment; TSVD: Total small vessel density; VA-ECMO: Venoarterial extracorporeal membrane oxygenation

\section{Acknowledgements}

The authors thank all participants of the National Taiwan University Hospital Center of Microcirculation Medical Research (NCMMR). We also thank Roger Lien (technician, MicroStar Instruments Co. Ltd., Taipei, Taiwan) for technical assistance in the microcirculation analysis.

\section{Funding}

This work was supported, in part, by a grant from the Taiwan Ministry of Science and Technology (MOST 104-2314-B-002-045) and a grant from the National Taiwan University Hospital (NTUH 105-A125).

\section{Availability of data and materials}

The datasets generated and/or analyzed during the current study are not publicly available due to the regulation of the Research Ethics Committee of National Taiwan University Hospital, but are available from the corresponding author on reasonable request.

\section{Authors' contributions}

YCY contributed to the design of the study, patient enrollment, interpretation of the data, writing of the manuscript, and revision of the manuscript. CTL contributed to the interpretation of the data and writing of the manuscript. CHW contributed to the design of the study, patient enrollment, interpretation of the data, and revision of the manuscript. YKT contributed to the statistics and interpretation of the data. $\mathrm{CHL}$ contributed to the design of the study and patient enrollment. YCW contributed to the interpretation of the data and revision of the manuscript. AC contributed to the design of the study. $\mathrm{CHH}$ contributed to the revision of the manuscript. YJC contributed to the design of the study and revision of the manuscript. YSC contributed to the design of the study and revision of the manuscript. All authors read and approved the final manuscript.

\section{Ethics approval and consent to participate}

This prospective observational study was approved by the Research Ethics Committee of National Taiwan University Hospital (approval number 201412045RINA).

\section{Consent for publication}

Informed consent of the patients was obtained from their legally authorized representatives before enrollment in the study.

\section{Competing interests}

The authors declare that they have no competing interests.

\section{Publisher's Note}

Springer Nature remains neutral with regard to jurisdictional claims in published maps and institutional affiliations.

\section{Author details}

'Department of Anesthesiology, National Taiwan University Hospital, College of Medicine, National Taiwan University, No 7, Chung Shang South Road, Taipei, Taiwan. ${ }^{2}$ Department of Surgery, National Taiwan University Hospital, College of Medicine, National Taiwan University, No 7, Chung Shang South Road, Taipei, Taiwan. ${ }^{3}$ Department of Public Health, Institute of Epidemiology \& Preventive Medicine, National Taiwan University, No. 17, Xu-Zhou Road, Taipei, Taiwan

\section{Received: 8 February 2018 Accepted: 22 May 2018}

Published online: 19 August 2018

\section{References}

1. Aissaoui N, Luyt CE, Leprince P, Trouillet JL, Leger P, Pavie A, Diebold B, Chastre J, Combes A. Predictors of successful extracorporeal membrane oxygenation (ECMO) weaning after assistance for refractory cardiogenic shock. Intensive Care Med. 2011;37(11):1738-45.
2. Chen YS, Lin JW, Yu HY, Ko WJ, Jerng JS, Chang WT, Chen WJ, Huang SC, Chi $\mathrm{NH}$, Wang $\mathrm{CH}$, et al. Cardiopulmonary resuscitation with assisted extracorporeal life-support versus conventional cardiopulmonary resuscitation in adults with in-hospital cardiac arrest: an observational study and propensity analysis. Lancet. 2008;372(9638):554-61.

3. Schwarz B, Mair P, Margreiter J, Pomaroli A, Hoermann C, Bonatti J, Lindner $\mathrm{KH}$. Experience with percutaneous venoarterial cardiopulmonary bypass for emergency circulatory support. Crit Care Med. 2003;31(3):758-64.

4. Chen YS, Chao A, Yu HY, Ko WJ, Wu IH, Chen RJ, Huang SC, Lin FY, Wang SS. Analysis and results of prolonged resuscitation in cardiac arrest patients rescued by extracorporeal membrane oxygenation. J Am Coll Cardiol. 2003; 41(2):197-203.

5. Yeh YC, Wang MJ, Chao A, Ko WJ, Chan WS, Fan SZ, Tsai JC, Sun WZ. Correlation between early sublingual small vessel density and late blood lactate level in critically ill surgical patients. J Surg Res. 2013;180(2):317-21.

6. Vellinga NA, Ince C, Boerma EC. Microvascular dysfunction in the surgical patient. Curr Opin Crit Care. 2010;16(4):377-83.

7. Jung C, Ferrari M, Rodiger C, Fritzenwanger M, Goebel B, Lauten A, Pfeifer R, Figulla HR. Evaluation of the sublingual microcirculation in cardiogenic shock. Clin Hemorheol Microcirc. 2009;42(2):141-8.

8. Boerma EC, Kuiper MA, Kingma WP, Egbers PH, Gerritsen RT, Ince C. Disparity between skin perfusion and sublingual microcirculatory alterations in severe sepsis and septic shock: a prospective observational study. Intensive Care Med. 2008;34(7):1294-8.

9. De Backer D, Creteur J, Preiser JC, Dubois MJ, Vincent JL. Microvascular blood flow is altered in patients with sepsis. Am J Respir Crit Care Med. 2002;166(1):98-104.

10. De Backer D, Donadello K, Sakr Y, Ospina-Tascon G, Salgado D, Scolletta S, Vincent JL. Microcirculatory alterations in patients with severe sepsis: impact of time of assessment and relationship with outcome. Crit Care Med. 2013; 41(3):791-9.

11. Trzeciak S, Dellinger RP, Parrillo JE, Guglielmi M, Bajaj J, Abate NL, Arnold RC, Colilla S, Zanotti S, Hollenberg SM. Early microcirculatory perfusion derangements in patients with severe sepsis and septic shock: relationship to hemodynamics, oxygen transport, and survival. Ann Emerg Med. 2007; 49(1):88-98.

12. van Genderen ME, Lima A, Akkerhuis M, Bakker J, van Bommel J. Persistent peripheral and microcirculatory perfusion alterations after out-of-hospital cardiac arrest are associated with poor survival. Crit Care Med. 2012;40(8):2287-94.

13. Kara A, Akin S, Dos Reis MD, Struijs A, Caliskan K, van Thiel RJ, Dubois EA, de Wilde W, Zijlstra F, Gommers D, et al. Microcirculatory assessment of patients under VA-ECMO. Crit Care. 2016;20(1):344.

14. Hutchings S, Watts S, Kirkman E. The Cytocam video microscope. A new method for visualising the microcirculation using incident dark field technology. Clin Hemorheol Microcirc. 2016;62(3):261-71.

15. Aykut G, Veenstra G, Scorcella C, Ince C, Boerma C. Cytocam-IDF (incident dark field illumination) imaging for bedside monitoring of the microcirculation. Intensive Care Med Exp. 2015;3(1):40.

16. Milstein DMJRE, Ince C. A novel computer-controlled high resolution video microscopy imaging system enables measuring mucosal subsurface focal depth for rapid acquisition of oral microcirculation video images. Intensive Care Med. 2012;38:S271

17. Huang SC, Yu HY, Ko WJ, Chen YS. Pressure criterion for placement of distal perfusion catheter to prevent limb ischemia during adult extracorporeal life support. J Thorac Cardiovasc Surg. 2004:128(5):776-7.

18. Ferreira FL, Bota DP, Bross A, Melot C, Vincent JL. Serial evaluation of the SOFA score to predict outcome in critically ill patients. JAMA. 2001;286(14): $1754-8$

19. Shore S, Nelson DP, Pearl JM, Manning PB, Wong H, Shanley TP, Keyser T, Schwartz SM. Usefulness of corticosteroid therapy in decreasing epinephrine requirements in critically ill infants with congenital heart disease. Am J Cardiol. 2001:88(5):591-4

20. De Backer D, Hollenberg S, Boerma C, Goedhart P, Buchele G, OspinaTascon G, Dobbe I, Ince C. How to evaluate the microcirculation: report of a round table conference. Crit Care. 2007;11(5):R101

21. Farquhar I, Martin CM, Lam C, Potter R, Ellis CG, Sibbald WJ. Decreased capillary density in vivo in bowel mucosa of rats with normotensive sepsis. Surg Res. 1996:61(1):190-6.

22. Sakr Y, Dubois MJ, De Backer D, Creteur J, Vincent JL. Persistent microcirculatory alterations are associated with organ failure and death in patients with septic shock. Crit Care Med. 2004;32(9):1825-31. 
23. Kara A, Akin S, Ince C. Monitoring microcirculation in critical illness. Curr Opin Crit Care. 2016;22(5):444-52.

24. Millar JE, Fanning JP, McDonald Cl, McAuley DF, Fraser JF. The inflammatory response to extracorporeal membrane oxygenation (ECMO): a review of the pathophysiology. Crit Care. 2016;20(1):387.

25. Sansone R, Stanske B, Keymel S, Schuler D, Horn P, Saeed D, Boeken U,

Westenfeld R, Lichtenberg A, Kelm M, et al. Macrovascular and

microvascular function after implantation of left ventricular assist devices in end-stage heart failure: role of microparticles. J Heart Lung Transplant. 2015: 34(7):921-32.

26. Sulkowski JP, Cooper JN, Pearson EG, Connelly JT, Rintoul N, Kilbaugh TJ, Deans KJ, Minneci PC. Hemolysis-associated nitric oxide dysregulation during extracorporeal membrane oxygenation. J Extra Corpor Technol. 2014 46(3):217-23.

27. Aissaoui N, El-Banayosy A, Combes A. How to wean a patient from venoarterial extracorporeal membrane oxygenation. Intensive Care Med. 2015; 41(5):902-5.

28. Luyt CE, Landivier A, Leprince P, Bernard M, Pavie A, Chastre J, Combes A. Usefulness of cardiac biomarkers to predict cardiac recovery in patients on extracorporeal membrane oxygenation support for refractory cardiogenic shock. J Crit Care. 2012;27(5):524.

Ready to submit your research? Choose BMC and benefit from:

- fast, convenient online submission

- thorough peer review by experienced researchers in your field

- rapid publication on acceptance

- support for research data, including large and complex data types

- gold Open Access which fosters wider collaboration and increased citations

- maximum visibility for your research: over $100 \mathrm{M}$ website views per year

At BMC, research is always in progress.

Learn more biomedcentral.com/submissions 